\title{
Mental Health of pneumoconiosis patients in China: a meta-analysis
}

\author{
Ruixue Huang*, Jian-An Hu* \\ Department of occupational and environmental Health, xiangya School of Public Health, Central South University.
}

Received: November 16, 2016; Accepted: December 30, 2016; Published: January 14, 2017

*Corresponding author: Jian-An Hu*, Department of occupational and environmental Health, School of Public Health, Central South University, 110 Xiangya Road, Changsha, 410078, China. Tel: 86-0731-84805460; Fax: 86-0731-84803006; E-mail: huja_xy@126.com

\begin{abstract}
Objective: To compare the mental health status of pneumoconiosis patients with that of healthy individuals in China with the aim of providing a scientific basis for psychological intervention and mental health education.

Methods: A computerized literature search was conducted using several databases, including CBM, Wan fang Data, VIP Information, CNKI, CMCI, and Pub Med, to collect articles published between 1999 and 2014 regarding the mental health of pneumoconiosis patients in China. The papers that met the selection criteria were subject to a meta-analysis, with RevMan 5.0 software used to process the data.
\end{abstract}

Results: A total of five articles involving 1342 cases met the research criteria and were selected for analysis. The meta-analysis revealed that, relative to healthy individuals, pneumoconiosis patients showed significantly more pronounced tendencies toward experiencing anxiety, depression, somatization, horror, forced, paranoia, interpersonal sensitivity, enmity, psychosis.

Conclusion: Serious mental health problems exist among pneumoconiosis patients in comparison with healthy individuals, particularly with regard to experiencing anxiety, depression. The mental health exhibited by pneumoconiosis patients appears to be worse than that of healthy individuals.

Keywords: pneumoconiosis patients; mental health; metaanalysis

\section{Introduction}

Pneumoconiosis is a systemic disease involving pulmonary interstitial fibrosis caused by long-term inhalation and retention of dust, generally as a result of occupational exposure. It has become one of the most serious occupational diseases, ranking as the highest with respect to incidence in China [1]. According to data from the Ministry of Health, nearly 700,000 cases of pneumoconiosis were reported prior to the end of 2010, resulting in nearly 150,000 deaths; this represents a high case fatality rate of $22.04 \%$ [2]. Given the long incubation, slow progression, irreversible deterioration of health, and complex complications that are associated with the disease, treatment of pneumoconiosis is generally focused on slowing the progression of the disease and prolonging survival time. Due to the lack of effective treatment measures, patients must endure great pain along with subsequent consequences for psychological health. As the medical paradigm has shifted from a predominantly biomedical model to biopsychosocial model, greater attention has been devoted to issues pertaining to psychological health during the medical treatment of pneumoconiosis.

For example, Zhang JF investigated symptoms of compulsion among 80 patients diagnosed with coal workers' pneumoconiosis (CWP). Their results showed that the incidence of obsessivecompulsive disorder among the pneumoconiosis patients (74.25\%) was significantly higher than that among the control group (42.00\%) [3]. Furthermore, Jiaxi Liao et al. investigated 312 cases of CWP using the Symptom Checklist (SCL-90) and found a high incidence of psychological disorders related to somatization, obsessive-compulsive symptoms, depression, anxiety, and hostility among those with CWP [4]. It is, therefore, important to study the psychological health status of CWP patients. Although there are many reports pertaining to the psychological health of CWP patients in China, research samples tend to be small, and numerous problems related to representativeness and consistency arise from the research samples, measurement tools, and statistical methods used in these studies. In the current study, we used a meta-analytic approach to conduct a more comprehensive and intuitive evaluation of a number of independent samples.

Meta-analysis is a statistical method that is used to compare and analyze results obtained by and reported in individual scientific studies. It uses statistical methods developed for the purpose of integrating the findings [5]. Hence, meta-analysis allows for the expansion of a pertinent sample, thereby leading to a more accurate interpretation of results. The current paper used a meta-analytic approach to assess the psychological health of CWP patients with the aim of providing information that may be useful in devising psychological interventions and improving the quality of life of these patients.

\section{Materials and methods}

All analyses were based on previously published studies; therefore, no ethical approval or patient consent was required. 
There are no conflicts of interest pertaining to the present article.

\section{Document sources}

Search strategy: A computer-based search of the following was conducted to collect all research published between 2000 and 2014 related to the psychological health of CWP patients: the Chinese Biomedical Literature Database (CBM), Articles database, VIP database, Chinese Academic Journal (CNKI), Pub Med, Chinese Biomedical Literature Database Journal (CMCI), and supplemental literature. The keywords were pneumoconiosis, psychological health, health condition, and quality of life. A total of 2677 documents were found, and 231 documents were included and analyzed after eliminating irrelevant or duplicate documents.

Selection of articles for analysis: The inclusion criteria for the articles were as follows:

1. the research sample included pneumoconiosis patients who met the national diagnostic criteria for CWP (diagnostic criteria: GB5906-1986, GB5906-1997, and GBZ70-2002.

2. The measurement tool used to assess psychological health was the SCL-90. 3. Each independent study used the standard scale of the SCL-90 to calculate means and standard deviations. For patients who underwent psychological intervention, data were obtained prior to treatment. Based on the above criteria, 231 documents were selected for further evaluation. Finally, five documents were included and analyzed such as duplicate reports, poor quality reports, reports with incomplete information, and studies in which the nonpneumoconiosis cases were represented by unqualified data.

\section{Statistical methods}

We checked the data and established a database in accordance with the requirements of the meta-analysis. We calculated the results using the classic meta-analysis method involving continuous variables. We selected the standardized mean difference (SMD) as the effect size for evaluating the difference between the pneumoconiosis and non-pneumoconiosis groups, and used the $X^{2}$ test as a statistical test of heterogeneity. The random effects model was selected based on the homogeneity test results. We then calculated the consolidated results of the standardized mean difference and 95\% confidence intervals (CIs). All analyses were conducted using the REVmAN5.2 software, as recommended by international evidence-based medical organizations.

All studies were approved by the Human Subjects Committees of Central South University.

\section{Results and analysis}

\section{Characteristics}

Based on the inclusion criteria, five studies with a total of 1342 cases, including 85 pneumoconiosis cases and 487 nonpneumoconiosis cases, were analyzed. The specific information pertaining to each document is shown in Table 1.

\section{Measurement results}

Consistency of results in each study: We used the random effects model to analyze the nine factors of the SCL-90; the results of the consistency test revealed significant heterogeneity $(\mathrm{p}<$ 0.0001).

Scores for each factor of the SCL-90: The results revealed significantly higher scores for the pneumoconiosis group relative to the non-pneumoconiosis group with respect to the following eight factors: somatization $(p=0.0002)$, forced $(p<0.0001)$, anxiety $(p=0.009)$, depression $(p<0.007)$, horror $(p=0.0004)$, psychosis $(p<0.0001)$, paranoia $(p<0.00001)$, interpersonal sensitivity $(p<0.0001)$ (Table 2$)$, enmity $(p=0.05)$.

\section{Analysis of forest plots}

The results of the meta-analysis indicate that both the horizontal lines and the diamond representing the effect size of the corresponding factor are located to the right of the line separating the two groups. This indicates that the mean average scores in the pneumoconiosis group were higher than they were in the non-pneumoconiosis group and that these differences were statistically significant (Figure 1).

\section{Analysis of publication bias}

Publication bias refers to the bias inherent in academic research, which favors the publication of some studies over others. Publication bias is of interest because literature reviews that are conducted to assess the strength of claims of

Table 1: Information pertaining to each document

\begin{tabular}{|c|c|c|c|c|c|c|c|}
\hline \multirow[b]{2}{*}{ NO. } & \multirow[b]{2}{*}{ Author } & \multirow[b]{2}{*}{ Date } & \multicolumn{3}{|c|}{$\mathbf{n}$} & \multirow[b]{2}{*}{ Area } & \multirow[b]{2}{*}{ Location } \\
\hline & & & $\begin{array}{c}\text { pneumoconiosis } \\
\text { cases }\end{array}$ & $\begin{array}{c}\text { non- } \\
\text { pneumoconiosis } \\
\text { patients }\end{array}$ & total & & \\
\hline 1 & Guixia Hou, et al. & 2003 & 78 & 50 & 128 & Coal pneumoconiosis & Taian \\
\hline 2 & Xiuying Kong, et al. & 2008 & 200 & 100 & 300 & Coal pneumoconiosis & Beijing \\
\hline 3 & Jiaxi Liao, et al. & 2011 & 312 & 122 & 434 & Coal pneumoconiosis & Sichuan \\
\hline 4 & Xinghui Li, et al. & 2011 & 117 & 83 & 200 & Coal pneumoconiosis & Ningxia \\
\hline 5 & Wenlin Xie, et al. & 2014 & 148 & 132 & 280 & $\begin{array}{l}\text { Coal pneumoconiosis, } \\
\text { silicosis et al }\end{array}$ & Zhejiang \\
\hline
\end{tabular}


support for a particular hypothesis, or of the values for a certain parameter, will themselves be biased if the original literature is contaminated by publication bias [6]. Although some criteria for publication are desirable (e.g., a bias against the publication of flawed studies), there is a tendency for researchers and journal editors to prefer certain outcomes over others (e.g., results reporting significant findings); this leads to a problematic bias in the published literature [7]. The funnel plot is designed to detect such publication bias. The scatter plot shows the included effects along the abscissa and the sample size or standard error of effect values along the vertical axis. If there were no publication bias, the data would be displayed as a symmetrical inverted funnel plot. The symmetry of funnel plot indicates that any publication bias present in the selected literature is negligible in its magnitude (Figure 2).

\section{Discussion}

Pneumoconiosis is one of the most important occupational diseases to occur in many countries, particularly in developing countries. Dust powder is the main culprit in China, where pneumoconiosis has become the most common occupational disease. Since the 1950s, a total of 749,970 cases of occupational disease have been reported in China. Of these, 676,541 are cases of pneumoconiosis; these have involved 527,431 patients and

Table 2 : Meta-analysis of the mental health of pneumoconiosis patients

\begin{tabular}{|c|c|c|c|c|}
\hline factor & $\mathbf{S M D}(\mathbf{9 5} \mathbf{9} \mathbf{C I})$ & $\mathbf{X}^{\mathbf{2}}$ & $\mathbf{Z}$ & $\boldsymbol{p}$ \\
\hline anxiety & $1.10(0.28,1.92)$ & 170.77 & 2.62 & 0.009 \\
\hline depression & $1.18(0.32,2.03)$ & 279.52 & 2.70 & 0.007 \\
\hline somatization & $1.03(0.48,1.59)$ & 80.80 & 3.67 & 0.0002 \\
\hline horror & $0.82(0.41,1.23)$ & 56.86 & 3.55 & 0.0004 \\
\hline forced & $0.82(0.41,1.23)$ & 46.79 & 3.90 & $<0.0001$ \\
\hline paranoia & $0.63(0.37,0.9)$ & 19.8 & 4.74 & $<0.00001$ \\
\hline $\begin{array}{c}\text { Interpersonal } \\
\text { sensitivity }\end{array}$ & $0.56(0.28,0.85)$ & 23.33 & 3.9 & $<0.0001$ \\
\hline enmity & $0.33(-0.00,0.66)$ & 32.28 & 1.96 & 0.05 \\
\hline psychosis & $0.77(0.55,1.00)$ & 14.28 & 6.72 & $<0.0001$ \\
\hline
\end{tabular}

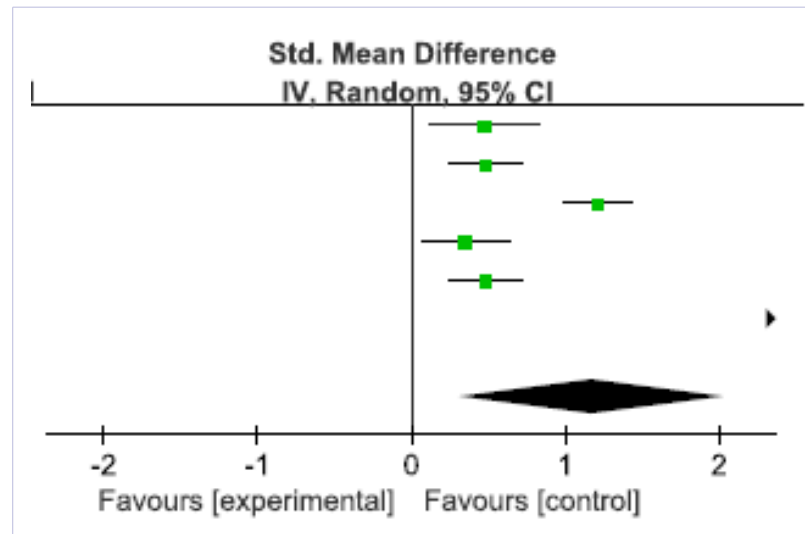

Figure 1: Forest plot of results

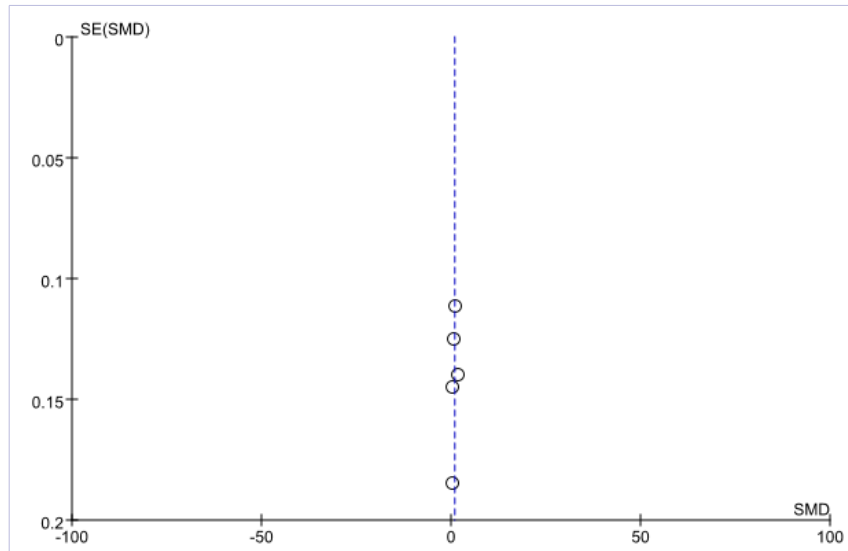

Figure 2: Funnel plot of results

have resulted in 149,110 deaths. The problems that confront pneumoconiosis patients, such as poor psychological health, economic hardship, and reduced quality of life, have drawn the attention of scholars, government officials, and society at large. [8]. Moreover, unexpected crises due to poor psychological health may occur, posing a potentially serious threat to patients. Many patients who suffer from pneumoconiosis occupy the foot of the social ladder and live under dire conditions and heavy economic hardship. They often experience guilt with respect to their family members and may be self-conscious, sensitive, suspicious and antisocial. Additionally, psychological problems such as anxiety and depression are common.

By applying a meta-analysis to numerous investigations conducted by diverse scholars, we were able to conduct a systematic and comprehensive quantitative evaluation of many independent research results obtained for the same research purpose to develop more comprehensive, intuitive, and reliable research conclusions. The results of our meta-analysis revealed statistical differences for all factors except hostility, anxiety, depression, somatization, horror, forced, paranoia, interpersonal sensitivity, enmity, psychosis, indicating that the mental health of pneumoconiosis patients was generally lower than that found in the non-pneumoconiosis group. The combined effect values for anxiety, depression, and somatization exceeded 1.00; therefore, these may be the principal factors responsible for the psychological problems of pneumoconiosis patients. Future mental health interventions for pneumoconiosis patients should address these dimensions in particular.

The SCL-90 scale, which is widely used abroad, was introduced in China in the 1980s and subsequently became widely used [9]. It is a popular self-evaluation scale, whose original design was intended to assess the symptoms of psychotic outpatients. In China, it was initially applied to research related to psychiatric symptoms. However, in addition to its application to psychiatric patients, the SCL-90 gradually came to be used with individuals deemed to be psychologically normal to objectively measure overall mental health rather than being limited to the assessment of the severity of psychiatric symptoms. Nonetheless, with respect to the contents of the scale, the SCL- 90 consists of a table 
of 90 symptoms and is used primarily to determine the presence and severity of certain psychological symptoms. Moreover, it has frequently been used as a screening tool by the Department of Psychiatry and the Consulting Outpatient Department (Qian Haiming); hence, it is not appropriate to consider it a test of the overall mental health of any specific group. Additionally, the SCL90 addresses only those symptoms that are experienced by an individual over a relatively short period of time (approximately 1 week), and responses are therefore likely to be affected by many factors. The typical pneumoconiosis patient experiences a long disease course, with persistent feelings of low selfesteem, hopelessness, hostility, anxiety, depression, and other symptoms. Future research should examine whether the SCL-90 is appropriate for assessing the mental health of pneumoconiosis patients.

The results of the meta-analysis reported in the present article integrate and strengthen the findings of recent research related to the psychological health of pneumoconiosis patients. However, it would be desirable to obtain data beyond those included in the five studies analyzed in the present research. Indeed, we were unable to consider a larger number of studies due to the inclusion criteria, the difficulty of publishing results with females that fail to reach statistical significance, and various other potential influences and biases that affect the available literature. Hence, additional repeated large-sample investigations would help to validate these results. Ideally, future research pertaining to the mental health of pneumoconiosis patients should aim to use a consistent research design and comprehensive methods of data analysis, thereby allowing for more reliable evaluation. Meanwhile, based on the results obtained in the present study, we recommend that government officials and health departments devote greater attention to the mental health of pneumoconiosis patients, strengthening the available psychological interventions for such patients and thereby improving their mental health.

\section{References}

1. Laney AS, Petsonk EL, Hale JM, Wolfe AL, Attfield MD. Potential determinants of coal workers' pneumoconiosis, advanced pneumoconiosis and progressive massive fibrosis among underground coal miners in the United States: 2005-2009. Am J Public Health. 2012; 102 Suppl 2:S279-83. Doi: 10.2105/AJPH.2011.300427..

2. Liu PC, Zhang D, Wu C. Quantity research of the CT in the coal pneumoconiosis. Zhong hua laodong Wei sheng zazhi .2002: 20:8893. (Chinese)

3. Zhang JF. Analysis of the compulsive symptom in coal pneumoconiosis. Occu Health 2008:24:2510-2512.

4. Liao JX, Yang CH, Zhang ZM. Mental disorders of coal workers with pneumoconiosis and social support. Zhongguo mental health zazhi .2011,19: 1339-1341. (Chinese)

5. Huang RX, Duan YY, Hu JA. Fish intake and liver cancer: a meta-analysis, PLoS One.2015; 8:5793 5797.

6. Li XH, Zao LF. Research on the psychological health status and quality of life in pneumoconiosis patients. Zhongguo laonian baojian yixue. 2011; 9: 29-30. (Chinese)

7. Guixia Hou. Study on the mental health among coal pneumoconiosis, China Occupationall Medicine2003:8:31-33.(Chinese)

8. Xiuying Kong. Study on the mental health among coal pneumoconiosis patients, China Occupational Medicine2008:7:35-37.(Chinese)

9. Weixin Xie. Study on the mental health among coal pneumoconiosis patients in Zhejiang Province. China Occupational Medicine2014:5:44-47.(Chinese) 